\title{
808 TERTIARY LYMPHOID STRUCTURE GENE SIGNATURE DETECTED IN IMMUNE CHECKPOINT INHIBITOR- ASSOCIATED RENAL IMMUNE RELATED ADVERSE EVENT
}

'Jamie Lin*, ${ }^{2}$ Amanda Tchakarov, ${ }^{1}$ Noha Abdel-Wahab, ${ }^{3}$ Houssein Safa, ${ }^{1}$ SalahEddine Bentebibel, ${ }^{4}$ Maen Abdelrahim, ${ }^{1}$ Cassian Yee, ${ }^{1}$ Adi Diab, ${ }^{1}$ Ala Abudayyeh. ${ }^{1}$ The University of Texas MD Anderson Cancer Center, Houston, TX, United States; ${ }^{2}$ University of Texas Health Science Center, Houston, TX, United States; ${ }^{3}$ Albert Einstein College of Medicine, Montefiore Medical Center, Bronx, NY, United States; ${ }^{4}$ Houston Methodist Cancer Center, Houston, TX, United States

Background Tertiary lymphoid structures (TLSs) have been previously associated with ICI induced response in patients with cancer, but a commensurate observation has not been made in ICI associated immune related adverse events (irAEs). Acute interstitial nephritis (AIN) is the predominant lesion reported in patients with renal irAEs, but various etiologies can also trigger the development of AIN including non-ICI drugs (e.g. non-steroidal anti-inflammatory drugs, antibiotics, proton pump inhibitors, etc.), and it is unknown whether these mechanisms are similar. With increasing indications for ICIs in cancer therapy, there is a critical need to define immune pathways driving the emergence of irAEs. To address this critical knowledge gap, we performed gene expression profiling on ICI-AIN, drug-AIN, and control (non-AIN) kidney biopsy specimens.

Methods Total RNA extracted from ICI-AIN ( $\mathrm{n}=6$ ), drugAIN ( $n=4)$, and control $(n=4)$ fixed formalin paraffin embedded archival kidney biopsy samples was analyzed by Nanostring nCounter PanCancer Immune Profiling Panel using NanoString nCounter FLEX Analysis System.

Results

Three comparisons were conducted: ICI-AIN vs control, drugAIN vs control, and ICI-AIN vs drug-AIN. A total of 147 genes were differentially expressed in ICI-AIN vs control and the most differentially expressed genes were CXCL 9, 10, and 11. Similarly, cell marker gene expression signatures (GES) revealed significant upregulation of $\mathrm{T}$ and $\mathrm{B}$ cell markers in ICI-AIN vs control $(\mathrm{P}<0.01)$ and ICI-AIN vs drug-AIN (T cell $\mathrm{P}<0.05 ; \mathrm{B}$ cell $\mathrm{P}<0.01)$. Differences in $\mathrm{T}$ and $\mathrm{B}$ cell score were not detected in drug-AIN vs control. Since irAEs have been associated with anti-tumor efficacy, we investigated whether a TLS signature could be detected in ICI-AIN using a four GES (CD79A, MS4A1, LAMP3 and POU2AF1). The ICIAIN group had significantly higher TLS score compared to both control and drug-AIN groups. Since several TLS signatures have been reported, we also calculated a 12-chemokine TLS GES which was also found to be statistically significant $(\mathrm{P}<0.05)$. Th1 and Th17 cells have been associated with the formation of TLS, differential upregulation of Th1 associated genes but not Th17 associated genes were detected. Furthermore, differential expression IFN-y and TNF signature was also observed in ICI-AIN group.

Conclusions This study is the first to demonstrate the presence of TLS immune signature in irAEs. Further investigations into the prognostic significance and strategies to uncouple ICI-associated anti-tumor benefits from ICI-induced irAEs should be explored.

Ethics Approval The study was approved by The University of Texas MD Anderson Cancer Center intuition's Ethics Board, approval number PA16-1016

http://dx.doi.org/10.1136/jitc-2021-SITC2021.808 